\title{
BMJ Open Timing of device reimplantation and reinfection rates following cardiac implantable electronic device infection: a systematic review and meta-analysis
}

\author{
Derek Chew, ${ }^{\oplus 1,2}$ Ranjani Somayaji, ${ }^{3,4,5,6}$ John Conly, ${ }^{3,4,5,6,7,8}$ Derek Exner, ${ }^{1,2,6}$ \\ Elissa Rennert-May ${ }^{3,4,6}$
}

To cite: Chew D, Somayaji R, Conly J, et al. Timing of device reimplantation and reinfection rates following cardiac implantable electronic device infection: a systematic review and meta-analysis. BMJ Open 2019;9:e029537. doi:10.1136/ bmjopen-2019-029537

- Prepublication history and additional material for this paper are available online. To view these files, please visit the journal online (http://dx.doi. org/10.1136/bmjopen-2019029537).

Received 01 February 2019 Revised 07 August 2019 Accepted 09 August 2019

Check for updates

(C) Author(s) (or their employer(s)) 2019. Re-use permitted under CC BY-NC. No commercial re-use. See rights and permissions. Published by BMJ.

For numbered affiliations see end of article.

Correspondence to Dr Elissa Rennert-May; elissa.rennertmay@ucalgary.ca

\section{ABSTRACT}

Objectives Initial management of cardiac implantable electronic device (CIED) infection requires removal of the infected CIED system and treatment with systemic antibiotics. However, the optimal timing to device reimplantation is unknown. The aim of this study was to quantify the incidence of reinfection after initial management of CIED infection, and to assess the effect of timing to reimplantation on reinfection rates.

Design Systematic review and meta-analysis.

Interventions A systematic review and meta-analysis was performed of studies published up to February 2018. Inclusion criteria were: (a) documented CIED infection, (b) studies that reported the timing to device reimplantation and (c) studies that reported the proportion of participants with device reinfection. A meta-analysis of proportions using a random effects model was performed to estimate the pooled device reinfection rate.

Primary and secondary outcome measures The primary outcome measure was the rate of CIED reinfection. The secondary outcome was all-cause mortality.

Results Of the 280 screened studies, 8 met inclusion criteria with an average of 96 participants per study (range 15-220 participants). The pooled incidence rate of device reinfection was $0.45 \%(95 \% \mathrm{Cl}, 0.02 \%$ to $1.23 \%)$ per person year. A longer time to device reimplantation $>72$ hours was associated with a trend towards higher rates of reinfection (unadjusted incident rate ratio 4.8; 95\% $\mathrm{Cl} 0.9$ to 24.3, $p=0.06$ ); however, the meta-regression analysis was unable to adjust for important clinical covariates. There did not appear to be a difference in reinfection rates when time to reimplantation was stratified at 1 week. Heterogeneity was moderate $\left(I^{2}=61 \%\right)$.

Conclusions The incident rate of reinfection following initial management of CIED infection is not insignificant. Time to reimplantation may affect subsequent rates of device reinfection. Our findings are considered exploratory and significant heterogeneity limits interpretation. PROSERO registration number CRD4201810960.

\section{INTRODUCTION}

Cardiac implantable electronic device (CIED) infections are a major cause of morbidity and mortality, ${ }^{12}$ and are associated with substantial healthcare costs. ${ }^{34}$ In the USA, one admission
Strengths and limitations of this study

- This is the first systematic review and meta-analysis to assess the rate of reinfection following initial cardiac implantable device infection.

- Pooled incidence rates of reinfection were obtained using random-effects meta-analysis of proportions model, and the impact of timing to device reimplantation was assessed by meta-regression.

- Substantial heterogeneity in the pooled incidence rates estimates limits interpretation.

- The results of our systematic review and meta-analysis highlight the need for additional well-designed studies in this area.

for an infected CIED can range from US $\$ 14$ 360 to US $\$ 53349 .^{3}$ As the number of CIED implantations increase worldwide, the rate of infectious complications is also rising. ${ }^{5-7}$ More concerningly, the rate of CIED infections is outpacing the increase in implantations. ${ }^{8}$ This is the likely result of the expanding indications for cardiac resynchronisation therapy (CRT) and prophylactic implantable cardioverter defibrillators (ICDs), and the increasing need for permanent pacing in an ageing patient population; CIEDs are being implanted in increasingly complex patients with multiple comorbidities who are at an increased risk of infectious complications. ${ }^{2}$ In addition, there is an increasing proportion of CIED surgeries for predicted battery depletion. ${ }^{5}$ Device pocket reintervention and repeat surgeries are known risk factors and increase the risk infection by more than two-fold. ${ }^{19}$

Treatment of CIED infections typically requires complete extraction of the infected CIED systems (including generator and leads), debridement and administration of antibiotics to eradicate infection. ${ }^{10}$ Delays in device extraction have been associated 
with significant increase in mortality. ${ }^{11}$ Furthermore, failure to remove an infected CIED has been associated with a sevenfold increase in 30-day mortality. ${ }^{12}$ There is also an increase in relapse of infection when hardware is not removed, ${ }^{13}$ which is postulated to be attributable to biofilm formation. ${ }^{14}$

Following CIED removal, it is critical to evaluate whether or not the CIED requires reimplantation, as over time the indication for CIED may no longer be present. ${ }^{10}$ In the majority of patients that require device replacement for infection, the optimal timing to reimplantation is unknown. The ideal reimplantation strategy would minimise the number of procedures and the duration of risk that patients may experience without a CIED (ie, ventricular arrhythmias, or worsening heart failure without resynchronisation). Notably, the optimal timing to reimplantation should not increase the risk of device reinfection.

The evidence regarding timing to reimplantation following CIED infection is relatively sparse and based primarily on consensus opinion. ${ }^{13} \mathrm{~A}$ recent prospective study assessed the relationship between timing of reimplantation to relapse of CIED infection within a 6-month time period. ${ }^{13}$ Their findings suggested that once the infected hardware was removed timing to reimplantation had little impact on recurrent infection. ${ }^{13}$

Current expert recommendations from the Heart Rhythm Society suggest that blood cultures should be negative for at least 72 hours prior to device reimplantation. ${ }^{10}$ A longer duration may be required depending on the clinical scenario (ie, if there is another untreated source of infection). In the presence of valvular vegetations, it is proposed that device reimplantation be delayed to a minimum of 14 days.

Given the relative paucity of evidence supporting the optimal timing to device reimplantation following CIED infection, our study aims to systematically review the available literature, to summarise the pooled reinfection rates after initial CIED infection, and to assess if there is a potential association of reinfection with time to device reimplantation.

\section{METHODS}

The study protocol and report is based on guidelines from the Preferred Reporting Items for Systematic Reviews and Meta-analyses statement ${ }^{15}$ and the Meta-Analysis of Observational Studies in Epidemiology (online supplementary appendix A). ${ }^{16}$ The study protocol was designed a priori and registered with PROSPERO.

\section{Eligibility criteria}

Publications were selected based on the following inclusion criteria: (a) cohort studies or randomised control trials that included patients with documented CIED infection with complete hardware removal as part of the management, (b) studies that reported the timing to device reimplantation following management of initial
CIED infection and (c) studies that reported the outcome of device reinfection following reimplantation. All publications were limited to those involving adult (age 18 years or older) human participants.

\section{Search strategy}

A systematic electronic search was performed in consultation with a librarian scientist, using MEDLINE (1946-), EMBASE (Excerpta Medica Database 1974-) and the Cochrane Library (including the Cochrane Database of Systematic Reviews, Database of Abstracts of Review Effects, Cochrane Central Registry of Controlled Trials, and Health Technology Assessment) databases for observational studies and randomised controlled trials (RCTs) that met inclusion criteria. This was followed with a hand search of the reference lists of relevant articles. The search strategy included both controlled vocabulary (Medical subject heading (MeSH) terms) as well as keywords that were identified during the scoping review. The main search concepts included (a) cardiac implantable devices, (b) device infection and (c) timing to device reimplantation. All searches were conducted without date limitations, and included manuscripts published up to 22 February 2018. The detailed search strategy is included in online supplementary appendix B.

\section{Study selection and data extraction}

Two reviewers (DC and ER-M) independently screened the study titles and abstracts to exclude irrelevant studies. The same reviewers then independently reviewed the full manuscripts for eligible studies and recorded the main reason for exclusion. Disagreements were resolved through consensus, and consultation of a third reviewer (RS) if necessary. Inter-rater agreement was quantified using Cohen's kappa coefficient. Data extraction was performed in duplicate by the same two reviewers into a standardised electronic spreadsheet. Data elements for extraction were pre-specified, and included the age and sex of participants, type of CIED infection (ie, endocarditis or pocket infection), device type (ie, pacemaker, ICD or CRT), number of leads, microbiology, timing to reimplantation, rate of device reinfection, mortality rate and study follow-up time.

\section{Assessment of study quality}

The risk of bias was assessed using the Newcastle-Ottawa Scale ${ }^{17}$ and updated Cochrane risk of bias tool ${ }^{18}$ for non-randomised controlled trials and RCTs, respectively. Risk of bias was assessed independently by two reviewers (DC and ER-M) and discrepancies resolved by consensus.

\section{Patient and public involvement}

Patients or members of the public were not involved in the study design or analysis.

\section{Statistical analysis}

The pooled incidence rate and 95\% CI for outcomes of interest were obtained. The primary outcome of the meta-analysis was the rate of CIED reinfection. The 
secondary outcome was all-cause mortality. Data were pooled using a random-effects meta-analysis of proportions model using the Dersimonian and Laird method ${ }^{19}$ incorporating a Freeman-Tukey double arcsine transformation..$^{20} \mathrm{~A}$ random-effects model was chosen a priori on the basis of the anticipated heterogeneity among study baseline characteristics and the impact on device reinfection rates. The Metaprop package in Stata IC V.15.1 (StataCorp, College Station, TX, USA) was used to obtain the pooled estimate. ${ }^{21}$ All analyses were performed using Stata IC V.15.1 with $p$ value $<0.05$ was considered to indicate statistical significance. Rates of CIED reinfection were standardised across studies by the duration of follow-up and reported as the incident rate per person year.

Due to the potential for significant variation in follow-up time and the possibility of zero count data in the included studies, we performed a secondary analysis using a mixed-effects Poisson-distribution model to estimate the pooled incidence rate of reinfection. ${ }^{22}$ To assess if reinfection rates were affected by time to device reimplantation, a meta-regression of the incidence rates and time to reimplantation was performed. Pre-specified subgroup analyses included stratification of the primary outcome by median time to device reimplantation of greater than 72 hours and those with a median reimplantation time of
72 hours or less. Reinfection rates stratified by reimplantation prior to 1 week versus at 1 week or greater were also assessed. The stratification of timing to reimplantation was chosen based on current expert recommendations for CIED infections without or with lead endocarditis, respectively. ${ }^{10} 23$

Heterogeneity across the studies was tested with the Cochran $\mathrm{Q}$ and $\mathrm{I}^{2}$ statistics. ${ }^{24}{ }^{25}$ We considered an $\mathrm{I}^{2}$ statistic of $>25 \%$ as a low degree of heterogeneity, $>50 \%$ as moderate heterogeneity and $>75 \%$ as high heterogeneity. ${ }^{26} \mathrm{~A}$ threshold of $\mathrm{p}<0.10$ was considered significant for the presence of heterogeneity.

\section{RESULTS}

\section{Study selection and characteristics of included studies}

Among 280 unique citations identified in the literature search, 18 studies were retrieved for full-text review (figure 1). The inter-rater agreement during the initial screening was substantial $(\kappa=0.61)$. Following the full-text review, a total of eight studies met inclusion criteria. ${ }^{13}$ 27-33 There were no RCTs identified in the systematic review that met the inclusion criteria. All eight included studies were observational in design; three were prospective $e^{132729}$ and five were retrospective. ${ }^{2830-33}$

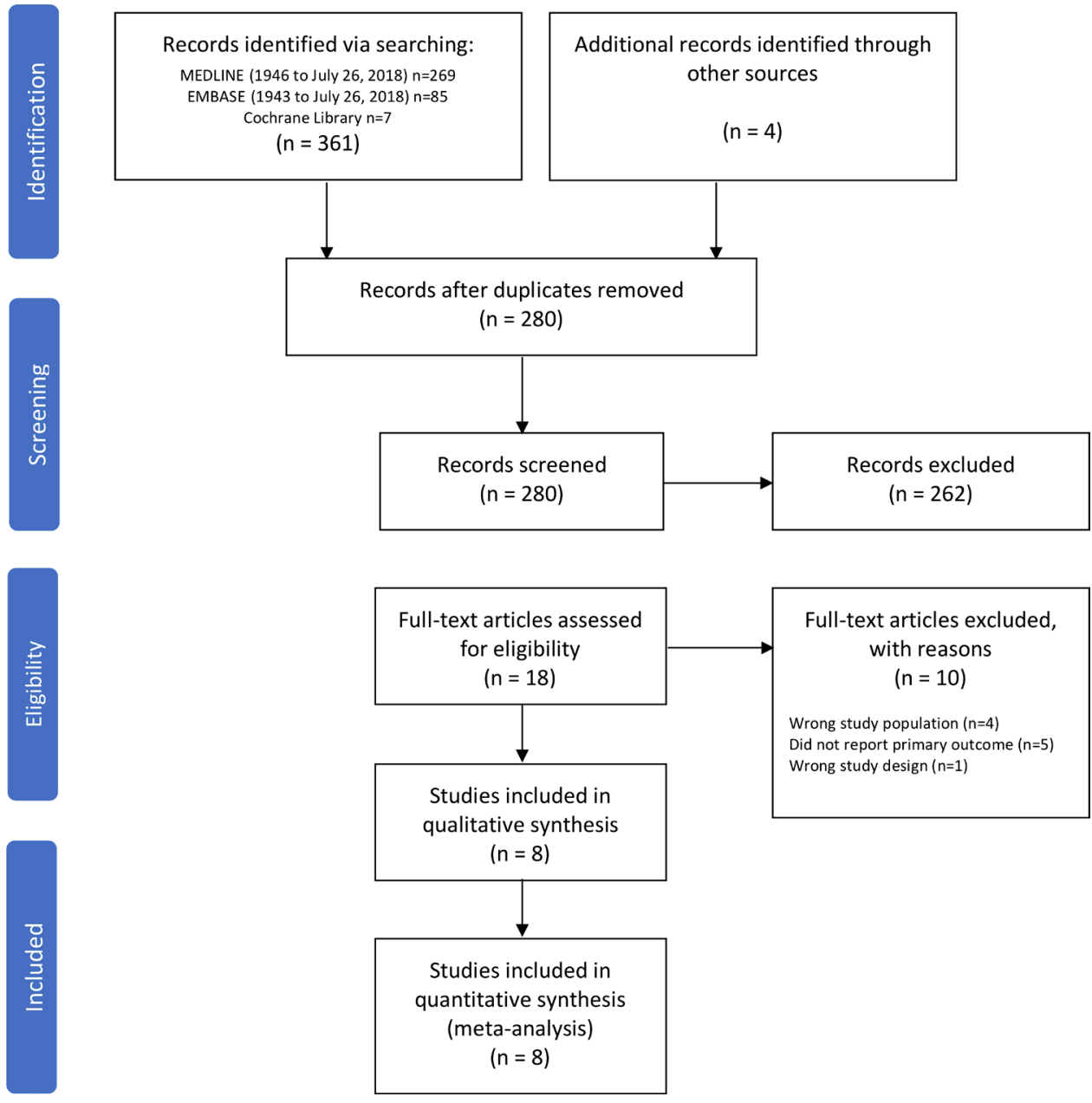

Figure 1 Flow diagram of study selection for systematic review. 
Of the ten studies that were excluded, four assessed the wrong study population, ${ }^{34-37}$ five did not report device reinfection rates, ${ }^{38-42}$ and one had the wrong study design. ${ }^{43}$

The characteristics of the included studies are summarised in table 1 . The observation cohorts had a range of 15-220 participants, for a total of 744 participants who underwent complete explantation of an infected CIED with subsequent reimplantation of a new device. The range of the mean age was 50-71 years and the proportion of women included in the studies was $19 \%-32 \%$. The majority of studies reported on device infections related to pacemakers and ICDs, ${ }^{27283133}$ and two studies included CRT infections. ${ }^{29} 32$ The microbiology of the CIED infections was not consistently reported among the studies. The most common reported aetiology of CIED infection was coagulase negative staphylococci (range 40\%-70\%), followed by Staphylococcus aureus (range 9\%-30\%) and Gram-negative bacilli (range 11\%-20\%). The duration of follow-up after device reimplantation varied substantially from 6 to 312 months.

\section{Study quality}

As there were no RCTs identified in the systematic review, the Newcastle-Ottawa Scale was used to assess risk of bias in each of the included studies (online supplementary appendix $\mathrm{C}$ ). Two measures within the scale were not applicable given the design of the included studies; the six measures that were graded were: (a) cohort representativeness, (b) exposure ascertainment, (c) outcome absence at baseline, (d) assessment of outcome, (e) adequacy of observation duration and (f) completeness of cohort follow-up. The majority of studies had the highest quality-level indicators in all six measures. Two studies selected populations that may not be representative of the overall CIED population: Mountantonakis $e t$ al focused on the patients with localised pocket infection for assessing the safety of same-day device reimplantation following extraction. ${ }^{31}$ The cohort described by Molina did not represent the contemporary population of CIED patients, as a significant proportion of devices in the study cohort underwent CIED implantation by sternotomy or thoracotomy with placement of epicardial patches. ${ }^{30}$ Two studies did not clearly detail if all patients had been accounted for by the end of the study and there were concerns of bias with regards to their completeness of follow-up. ${ }^{30} 33$

\section{Reinfection following management of infection CIED infection}

In our primary analysis, the incidence rate of first device reinfection for the pooled cohort was $0.45 \%$ (95\% CI, $0.02 \%$ to $1.23 \%$ ) per person year (figure 2 ). There was a moderate degree of heterogeneity $\left(I^{2}=61 \%\right.$, Cochran's $Q$ $\mathrm{p}=0.01$ ). In our secondary analysis using a mixed-effects Poisson regression model, the incidence rate of reinfection was similar to our primary analysis at $0.58 \%(95 \% \mathrm{CI}$, $0.21 \%$ to $1.55 \%$ ) per person year.

\section{Effect of time to reimplantation}

Time to reimplantation $>72$ hours was associated with a trend towards a higher incidence of CIED reinfection (unadjusted incident rate ratio $4.8 ; 95 \%$ CI 0.9 to 24.3, $\mathrm{p}=0.06$ mixed-effects Poisson regression). Given the smaller number of included studies identified by the systematic review, we were unable to adjust for additional variables. When stratifying time to device reimplantation by 1 week or less, there did not appear to be a significant difference in incidence rate $(p=0.7$, mixed-effects Poisson regression).

\section{Mortality following CIED infection}

Only five of the included studies reported all-cause mortality. ${ }^{1327-30}$ Among the 508 patients included in these studies, 42 deaths $(8.3 \%)$ were observed over a median follow-up of 14 months. In the random-effects analysis, the incidence rate of death was $5.0 \%(95 \%$ CI, $0.1 \%$ to $15.5 \%$ ) per person year of follow-up (figure 3). There was a high degree of heterogeneity $\left(I^{2}=96 \%\right.$, Cochran's $\mathrm{Q} p<0.001$ ).

\section{DISCUSSION}

\section{Principle findings}

We found that the pooled reinfection rate following initial management of CIED infection was approximately $0.5 \%$ per person-year. To our knowledge, this is the first systematic review and meta-analysis reporting the pooled reinfection rates following original management of CIED infection. The substantial heterogeneity seen in the pooled analysis suggests the presence of several variables that can affect the incidence rate of reinfection. Factors may include the presence of bacteraemia, response to treatment, or patient factors, such as the presence of immunosuppression. ${ }^{1}$

When we examined infection risk based on timing of reimplantation, a time of greater than 72 hours was associated with a fourfold higher incidence rate compared with reimplantation at 72 hours or less. Using a 1 week cut-point for time to device reimplantation, there was no difference in reinfection rates. We consider these results exploratory, as meta-regression is considered an underpowered analysis. Specifically, given the small number of studies included in the pooled analysis, we were unable to adjust for potentially important confounders. For example, the higher reinfection rate associated with time to reimplantation $>72$ hours may be due to an increased number of comorbid conditions in the corresponding study populations, or a high proportion of systemic infections requiring additional time to clear the bloodstream of bacteraemia. Important covariates, which we were unable to adjust for at the meta-regression level, were the proportions of documented endocarditis, lead vegetations or bacteraemia compared with localised pocket infection.

\section{Management of cardiac device infections}

Treatment of CIED infections requires complete extraction of the infected CIED systems (including 


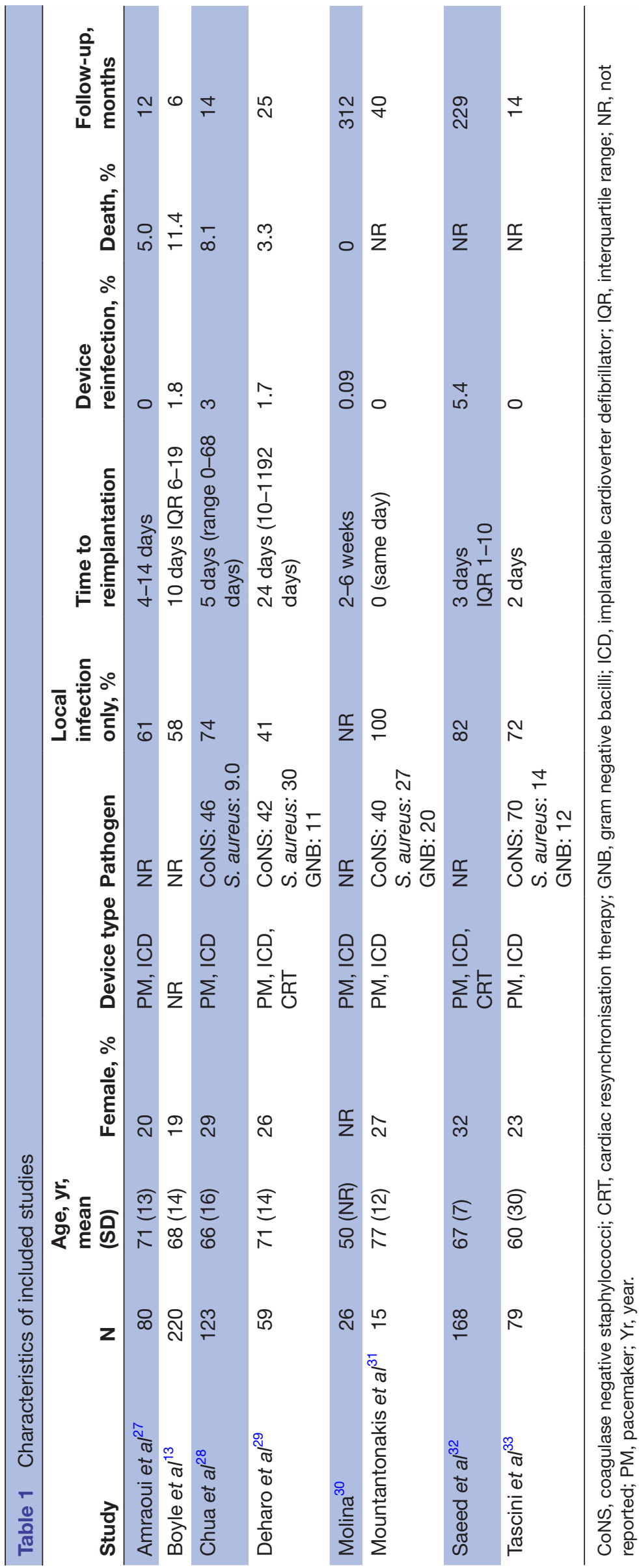

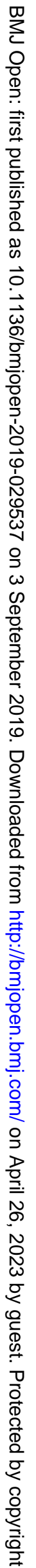


Study

ES $(95 \% \mathrm{Cl}), \% \quad$ Weight , $\%$

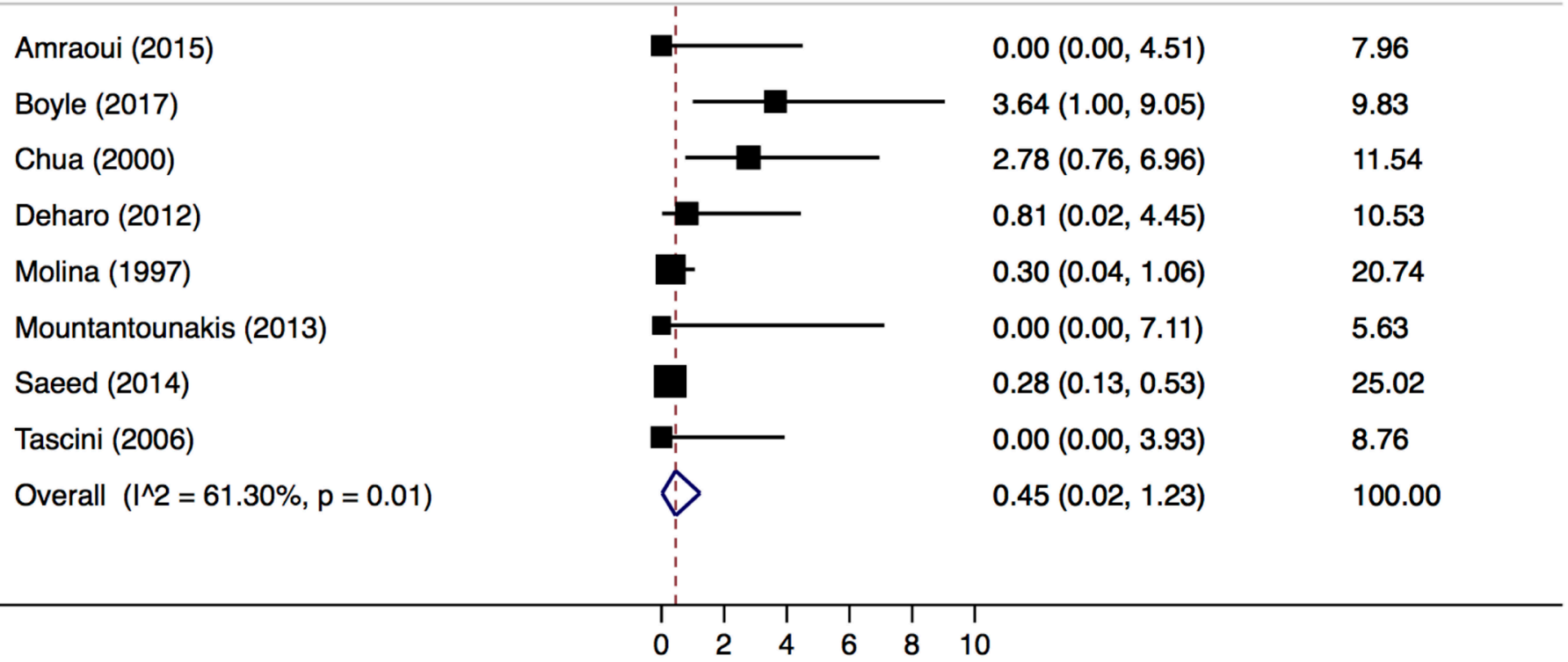

Incidence Rate of Reinfection, \% per person year

Figure 2 Pooled incidence rate of device reinfection. ES; effect size, Cl; confidence interval.

generator and leads) and administration of systemic antibiotics to eradicate infection. ${ }^{10}$ In the majority of patients that require device replacement, the optimal timing to reimplantation is unknown. In our study, there was an unexpected association of increased reinfection rates with a time to device reimplantation greater than 72 hours. Although interpretation is limited by lack of adjustment for confounders, this finding is opposite to the expectation in clinical practice. Conceptually, reimplanting a device too early during the treatment course may result in a higher infection relapse rate if the infection has only been partially eradicated by systemic antibiotics. On the other hand, the longer hospital stays while awaiting device reimplantation are associated with increased healthcare costs, decreased patient quality of life and the potential for acquiring non-device related nosocomial infections. Furthermore, there is the potential for adverse events related to the absence of ICD or CRT therapies such as worsening heart failure or delay to treatment of malignant ventricular arrhythmias.

\section{Time to device reimplantation}

There is a paucity in the literature exploring the timing of CIED replacement and risk of reinfection.

Study

$$
\text { ES }(95 \% \mathrm{Cl}), \% \quad \text { Weight, } \%
$$

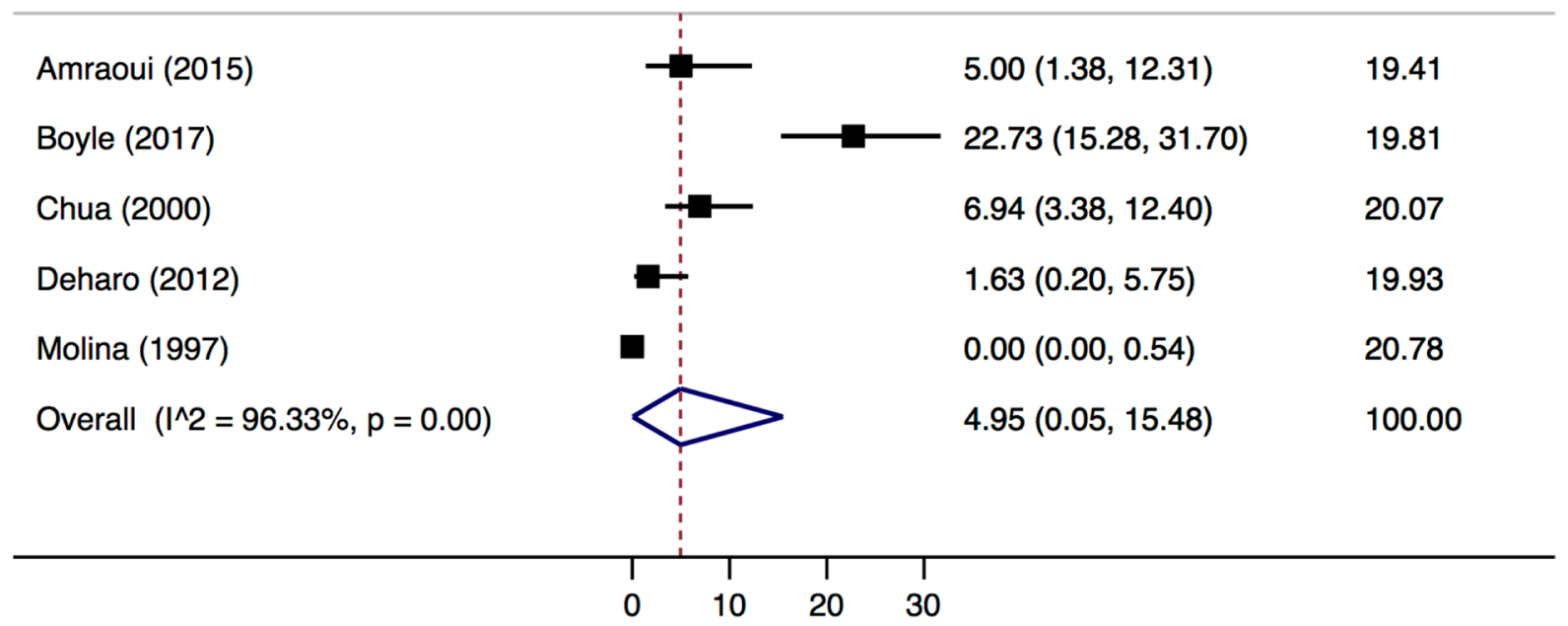

Incidence Rate of Death, \% per person year

Figure 3 Pooled incidence rate of death following CIED infection. ES; effect size, Cl; confidence interval. 
Our systematic review only identified eight studies that reported the time to reimplantation and the rate of device reinfection. ${ }^{13}$ 27-33 The majority of these studies are limited by their retrospective study design ${ }^{28}{ }^{30-33}$ and small sample sizes. Furthermore, the primary study designs did not focus on assessing the effect of time to reimplantation on subsequent CIED reinfections. Consistent with the relatively paucity in the literature guiding time to device reimplantation, we noted a wide range in the median time to device reimplantation (ie, same-day reimplantation to over 2 weeks).

One of the largest contemporary prospective cohorts tracking CIED infections found a repeat infection risk of $1.8 \%$ among patients who were reimplanted. ${ }^{13}$ This study found a high variation in physician practice when determining the time to device reimplantation; yet timing to reimplantation did not seem to affect reinfection rates. With the caveat of a relatively small sample size and limited follow-up, the authors suggested that an array of other risk factors may have a larger role in determining infection relapse rates compared with decisions regarding timing to reimplantation. ${ }^{13}$ Consistent with this notion, some studies suggest that factors such as the presence of haemodialysis for renal failure, pocket haematomas, malignancy or $S$. aureus bacteraemia are the leading risk factors in estimating the risk of reinfection. ${ }^{45}$ In fact, small single-centre studies suggested that same-day reimplantation is feasible for patients with isolated CIED pocket infections and is not associated with adverse outcomes. ${ }^{3146}$

Current expert consensus recommendations from the Heart Rhythm Society and American Heart Association suggest a 72-hour and 14-day waiting period for reimplantation based on blood culture negativity and the presence of valvular vegetations, respectively. ${ }^{10}{ }^{23}$ However, the quality of evidence supporting these recommendations are weak (Grade IIaC), and based mainly on a single retrospective study of 127 patients. ${ }^{42}$ Our findings do not support this conclusion as reimplantation at greater than 72 hours was associated with increased infection rates. However, there are significant limitations to our findings since only eight studies were available for inclusion in the meta-regression, and these individual studies did not reliably report differences in patient characteristics among those who developed a device reinfection versus those who remained infection-free. Thus, we were unable to adjust for important covariates such as severity of initial infection (ie, presence of bacteraemia, lead endocarditis, causative micro-organism), patient comorbidities or choice of antibiotic treatment for initial infection. Our meta-regression findings should be considered exploratory and reinforces the need for additional research to guide recommendations regarding timing to reimplantation.

\section{Study limitations}

Our study requires interpretation in the context of a number of limitations. First, time to reimplantation and device reinfection rates were inconsistently reported in the literature. Five studies were excluded at the level of the full-text screen as they did not report device reinfection rates. In addition, the adopted diagnostic criteria for device infection were inconsistently reported among the included studies, which may contribute to the heterogeneity in the pooled estimate of incidence rate of CIED reinfection. Second, the meta-analyses included mainly retrospective studies that varied in patient population, study quality and follow-up, contributing to the clinical variability and heterogeneity in our pooled analysis. Third, we did not anticipate the relatively small number of studies and patients derived from the systematic review. Nonetheless, our study highlights the importance of additional research in the area of cardiac device infection, and further study assessing reinfection rates and long-term outcome.

Finally, we attempted to explore the relationship between time to reimplantation and reinfection rates. An important limitation is the unavailability of patientlevel data and times to follow-up. To explore the potential association, we performed a Poisson-distribution meta-regression. However, given the small number of studies that met inclusion criteria, the meta-regression was underpowered, and we were unable to properly adjust for other confounders with the potential to affect reinfection rates. This may explain the unexpected association of increased reinfection rates with time to device reimplantation greater than 72 hours. Nevertheless, this highlights the need for larger prospective studies to adequate control for confounders when exploring reinfection risk after initial CIED infection.

\section{Implications for future research}

Additional prospective, well-designed studies are required to explore the effect of timing to reimplantation on reinfection rates, with adequate adjustment for patient comorbidities, extent of infection (ie, localised pocket infection, vegetation or bacteraemia) and the causative pathogen. Based on the several small studies reporting on the safety of same-day reimplantation and in light of our findings, larger studies are necessary to validate the safety of the one-stage contralateral device replacement approach compared with delayed device replacement. Given the potential impact on hospital length of stay, an economic evaluation comparing these strategies will also be an important component.

Finally, the advent of new technology such as leadless pacemakers and subcutaneous ICDs may obviate the need to delay device reimplantation following extraction of infected CIED systems. The current assumption is that these newer devices are associated with a lower risk of infection: leadless pacemakers have significantly less surface area for bacterial seeding, and subcutaneous ICDs do not contain any components 
exposed to the bloodstream. Nevertheless, the use of these novel devices to replace infected conventional CIEDs following antimicrobial therapy, or the rates of infection associated with these devices have yet to be assessed.

\section{CONCLUSION}

The incident rate of reinfection following initial management of CIED infection is not insignificant. Our findings suggest a trend that time to reimplantation affects rates of reinfection when device reimplantation occurs at $\leq 72$ hours compared with $>72$ hours. The findings of this study need to be interpreted with circumspection due to the moderate heterogeneity among included studies.

\section{Author affiliations}

${ }^{1}$ Department of Cardiac Sciences, University of Calgary, Calgary, Alberta, Canada ${ }^{2}$ Libin Cardiovascular Institute of Alberta, University of Calgary, Calgary, Alberta, Canada

${ }^{3}$ Department of Medicine, University of Calgary, Calgary, Alberta, Canada ${ }^{4} 0$ 'Brien Institute for Public Health, University of Calgary, Calgary, Alberta, Canada ${ }^{5}$ Snyder Institute for Chronic Diseases, University of Calgary, Calgary, Alberta, Canada

${ }^{6}$ Department of Community Health Sciences, University of Calgary, Calgary, Alberta, Canada

${ }^{7}$ Department of Microbiology, Immunology and Infectious Diseases, University of Calgary, Calgary, Alberta, Canada

${ }^{8}$ Department of Pathology \& Laboratory Medicine, University of Calgary, Calgary, Alberta, Canada

Acknowledgements We would like to thank Dr Diane Lorenzetti, PhD (University of Calgary librarian scientist) for her assistance with refining the systematic search strategy.

Contributors JC, DE, ER-M and DC were responsible for conception of this research study and methodology. DS and ER-M wrote the initial draft of the manuscript. DS, ER-M and RS were responsible for data collection and analysis. All authors reviewed the manuscript and analyses.

Funding DC is supported by an Arthur J.E. Child Cardiology Fellowship and a Banting Postdoctoral Fellowship. DE is supported by a Government of Canada Tier 1 Research Chair in Cardiovascular Clinical Trials, the Canadian Arrhythmia Network of Canada and a Canadian Institutes of Health Research Operating grant. ER-M is supported by an Alberta Innovates Clinician Fellowship. RS is supported by research grants from the Cystic Fibrosis Foundation, Cystic Fibrosis Canada and the Canadian Institutes for Health Research.

Competing interests DE has received research grants from Medtronic, GE Healthcare and St Jude Medical outside of the submitted work.

Patient consent for publication Not required.

Provenance and peer review Not commissioned; externally peer reviewed.

Data availability statement Data are available upon reasonable request.

Open access This is an open access article distributed in accordance with the Creative Commons Attribution Non Commercial (CC BY-NC 4.0) license, which permits others to distribute, remix, adapt, build upon this work non-commercially, and license their derivative works on different terms, provided the original work is properly cited, appropriate credit is given, any changes made indicated, and the use is non-commercial. See: http://creativecommons.org/licenses/by-nc/4.0/.

\section{REFERENCES}

1. Polyzos KA, Konstantelias AA, Falagas ME. Risk factors for cardiac implantable electronic device infection: a systematic review and meta-analysis. Europace 2015;17:767-77.
2. Greenspon AJ, Patel JD, Lau E, et al. 16-Year trends in the infection burden for pacemakers and implantable cardioverter-defibrillators in the United States 1993 to 2008. J Am Coll Cardiol 2011;58:1001-6.

3. Sohail MR, Henrikson CA, Braid-Forbes MJ, et al. Mortality and cost associated with cardiovascular implantable electronic device infections. Arch Intern Med 2011;171:1821-8.

4. Tarakji KG, Chan EJ, Cantillon DJ, et al. Cardiac implantable electronic device infections: presentation, management, and patient outcomes. Heart Rhythm 2010;7:1043-7.

5. Mond HG, Proclemer A. The 11th world survey of cardiac pacing and implantable cardioverter-defibrillators: calendar year 2009-a World Society of Arrhythmia's project. Pacing Clin Electrophysiol 2011;34:1013-27.

6. Valzania C, Torbica A, Tarricone R, et al. Implant rates of cardiac implantable electrical devices in Europe: a systematic literature review. Health Policy 2016;120:1-15.

7. Raatikainen MJP, Arnar DO, Merkely B, et al. A decade of information on the use of cardiac implantable electronic devices and interventional electrophysiological procedures in the European Society of cardiology countries: 2017 report from the European heart rhythm association. Europace 2017; 19(suppl_2):ii1-ii90

8. Voigt A, Shalaby A, Saba S. Continued rise in rates of cardiovascular implantable electronic device infections in the United States: temporal trends and causative insights. Pacing Clin Electrophysiol 2010;33:414-9.

9. Arana-Rueda E, Pedrote A, Frutos-López M, et al. Repeated procedures at the generator pocket are a determinant of implantable cardioverter-defibrillator infection. Clin Cardiol 2017;40:892-8.

10. Kusumoto FM, Schoenfeld MH, Wilkoff BL, et al. $2017 \mathrm{Hrs}$ expert consensus statement on cardiovascular implantable electronic device lead management and extraction. Heart Rhythm 2017; 14:e503-51.

11. Viganego F, O'Donoghue S, Eldadah Z, et al. Effect of early diagnosis and treatment with percutaneous lead extraction on survival in patients with cardiac device infections. Am J Cardiol 2012;109:1466-71.

12. Le KY, Sohail MR, Friedman PA, et al. Impact of timing of device removal on mortality in patients with cardiovascular implantable electronic device infections. Heart Rhythm 2011;8:1678-85.

13. Boyle TA, Uslan DZ, Prutkin JM, et al. Reimplantation and repeat infection after Cardiac-Implantable electronic device infections: experience from the medic (multicenter electrophysiologic device infection cohort) database. Circ Arrhythm Electrophysiol 2017;10.

14. Gaynor SL, Zierer A, Lawton JS, et al. Laser assistance for extraction of chronically implanted endocardial leads: infectious versus noninfectious indications. Pacing Clin Electro 2006;29:1352-8.

15. Moher D, Liberati A, Tetzlaff J, et al. Preferred reporting items for systematic reviews and meta-analyses: the PRISMA statement. BMJ 2009;339:b2535.

16. Stroup DF, Berlin JA, Morton SC, et al. Meta-analysis of observational studies in epidemiology: a proposal for reporting. meta-analysis of observational studies in epidemiology (moose) group. JAMA 2000;283:2008-12.

17. Wells GA. The Newcastle-Ottawa Scale (NOS) for assessing the quality of nonrandomised studies in meta-analyses, 2000. Available: <http://www.ohri.ca/programs/clinical_epidemiology/oxford.asp]>

18. Higgins JP, Chandler J, McKenzie I, et al. Cochrane methods. CDSR 2016;10 (Suppl 1).

19. DerSimonian R, Laird N. Meta-analysis in clinical trials. Control Clin Trials 1986;7:177-88.

20. Freeman MF, Tukey JW. Transformations related to the angular and the square root. Ann Math Statist 1950;21:607-11.

21. Nyaga VN, Arbyn M, Aerts M. Metaprop: a Stata command to perform meta-analysis of binomial data. Arch Public Health 2014;72:39.

22. Spittal MJ, Pirkis J, Gurrin LC. Meta-analysis of incidence rate data in the presence of zero events. BMC Med Res Methodol 2015;15:42.

23. Baddour LM, Epstein AE, Erickson CC, et al. Update on cardiovascular implantable electronic device infections and their management: a scientific statement from the American heart association. Circulation 2010;121:458-77.

24. Cochran WG. The comparison of percentages in matched samples. Biometrika 1950;37:256-66.

25. Higgins JPT, Thompson SG. Quantifying heterogeneity in a metaanalysis. Stat Med 2002;21:1539-58.

26. Higgins JPT, Thompson SG, Deeks JJ. Measuring inconsistency in meta-analyses. BMJ 2003;327:557-60.

27. Amraoui S, Sohal M, Li A, et al. Comparison of delayed transvenous reimplantation and immediate surgical epicardial approach in pacingdependent patients undergoing extraction of infected permanent pacemakers. Heart Rhythm 2015;12:1209-15. 
28. Chua JD, Wilkoff BL, Lee I, et al. Diagnosis and management of infections involving implantable electrophysiologic cardiac devices. Ann Intern Med 2000;133:604-8.

29. Deharo J-C, Quatre A, Mancini J, et al. Long-term outcomes following infection of cardiac implantable electronic devices: a prospective matched cohort study. Heart 2012;98:724-31.

30. Molina JE. Undertreatment and overtreatment of patients with infected antiarrhythmic implantable devices. Ann Thorac Surg 1997;63:504-9.

31. Mountantonakis SE, Tschabrunn CM, Deyell MW, et al. Same-day contralateral implantation of a permanent device after lead extraction for isolated pocket infection. Europace 2014;16:252-7.

32. Saeed O, Gupta A, Gross JN, et al. Rate of cardiovascular implantable electronic device (CIED) re-extraction after recurrent infection. Pacing Clin Electrophysiol 2014;37:963-8.

33. Tascini C, Bongiorni MG, Gemignani G, et al. Management of cardiac device infections: a retrospective survey of a non-surgical approach combining antibiotic therapy with transvenous removal. J Chemother 2006;18:157-63.

34. Amraoui S, Barandon L, Whinnett Z, et al. Single surgical procedure combining epicardial pacemaker implantation and subsequent extraction of the infected pacing system for pacemaker-dependent patients. J Thorac Cardiovasc Surg 2013;146:302-5.

35. Le Franc P, Klug D, Jarwe M, et al. Extraction of endocardial implantable cardioverter-defibrillator leads. Am J Cardiol 1999;84:187-91.

36. O'Nunain S, Perez I, Roelke M, et al. The treatment of patients with infected implantable cardioverter-defibrillator systems. J Thorac Cardiovasc Surg 1997;113:121-9.
37. Rickard J, Tarakji K, Cronin E, et al. Cardiac venous left ventricular lead removal and reimplantation following device infection: a large single-center experience. J Cardiovasc Electrophysiol 2012;23:1213-6.

38. Cassagneau R, Ploux S, Ritter P, et al. Long-term outcomes after pocket or scar revision and reimplantation of pacemakers with preerosion. Pacing Clin Electrophysiol 2011;34:150-4.

39. Kennelly BM, Piller LW. Management of infected transvenous permanent pacemakers. Heart 1974;36:1133-40.

40. Margey R, McCann H, Blake G, et al. Contemporary management of and outcomes from cardiac device related infections. Europace 2010;12:64-70

41. Rickard J, Tarakji K, Cheng A, et al. Survival of patients with biventricular devices after device infection, extraction, and reimplantation. JACC Heart Fail 2013;1:508-13.

42. Sohail MR, Uslan DZ, Khan AH, et al. Management and outcome of permanent pacemaker and implantable cardioverter-defibrillator infections. J Am Coll Cardiol 2007:49:1851-9.

43. Vogt PR, Sagdic K, Lachat M, et al. Surgical management of infected permanent transvenous pacemaker systems: ten year experience. J Card Surg 1996;11:180-6.

44. Essebag V, Verma A, Healey JS, et al. Clinically significant pocket hematoma increases long-term risk of device infection: bruise contro infection study. J Am Coll Cardiol 2016;67:1300-8.

45. Leung S, Danik S. Prevention, diagnosis, and treatment of cardiac implantable electronic device infections. Curr Cardiol Rep 2016;18:58

46. Nandyala R, Parsonnet V. One stage side-to-side replacement of infected pulse generators and leads. Pacing Clin Electrophysiol 2006;29:393-6. 